\title{
A LANGUAGE FOR Ontological NiHilism
}

\author{
CATHARINE DIEHL \\ University of Toronto
}

\begin{abstract}
According to ontological nihilism there are, fundamentally, no individuals. Both natural languages and standard predicate logic, however, appear to be committed to a picture of the world as containing individual objects. This leads to what I call the expressibility challenge for ontological nihilism: what language can the ontological nihilist use to express her account of how matters fundamentally stand? One promising suggestion is for the nihilist to use a form of predicate functorese, a language developed by Quine. This proposal faces a difficult objection, according to which any theory in predicate functorese will be a notational variant of the corresponding theory stated in standard predicate logic. Jason Turner (2011) has provided the most detailed and convincing version of this objection. In the present paper, I argue that Turner's case for the notational variance thesis relies on a faulty metasemantic principle and, consequently, that an objection long thought devastating is in fact misguided.
\end{abstract}

$\mathrm{B}$ отн standard metaphysics and common sense are, plausibly, committed to a picture of the world as containing individual objects: these objects range from the mid-sized dry goods of everyday life such as trees, tables, and turnips to electrons, protons, and neutrons. These objects belong to kinds-biological, chemical, physical, etc.-but they are particular instances of these kinds. It is these particular objects that we seem to encounter in perception, and they are central to much of our ordinary communication about the world. Viewing the world as containing concrete, particular objects-henceforth 'individuals' - is so fundamental to our cognitive operations that the project of devising a metaphysics without individuals might seem hopeless. Recently, however, several philosophers have challenged this picture, motivated by puzzles stemming from both metaphysics and (a certain interpretation of) the findings of physics. They argue that contrary to appearances, fundamental reality does not include any individual

Contact: Catharine Diehl <catharine.diehl@utoronto.ca> 
object of any kind. ${ }^{1}$ Instead, all fundamental matters of fact are 'general' - a view I call 'ontological nihilism,' following Hawthorne and Cortens (1995) and Turner (2011)

While ontological nihilism denies that the world fundamentally includes individuals, it does hold that the world has a determinate nature. But what, according to the nihilist, is the character of this nature? The pervasiveness of thing-talk in natural language makes it hard for the ontological nihilist to express her picture of "the way the world is. ${ }^{2}$ While the ontological nihilist does not think that there are objects, she also does not hold that there is simply, in Jason Turner's phrase, a "blob ... without the blob" (Turner 2011: 4). Since natural and standard formal languages do not suffice for expressing a nihilistic picture of the world, the nihilist must propose alternative linguistic resources in which to state her account of the world. Thing-talk provides the individualist with a ready-made way to express her views, which the nihilist lacks. The nihilist needs to develop a language that can perspicuously express her view of the fundamental character of the world. ${ }^{3}$ I shall call the problem of devising such linguistic resources the expressibility challenge for ontological nihilism.

One promising response to the expressibility challenge is to use predicate functorese (PF), a formal system developed by Quine, as a substitute for classical predicate logic and to provide PF with an interpretation using a metaphysics of features - non-objectual states of affairs modeled on what is attributed by claims such as 'It is raining' or 'It is cold.' ${ }^{4}$ The most prominent and long-standing challenge faced by PF-nihilism is the objection from notational variance, according to which any theory in PF must have the same ontological commitments as the

1. See Dasgupta (2009), Hawthorne and Cortens (1995), Ketland (2015), and Russell (2018). There is a tricky issue here concerning the role of fundamentality in the nihilist's thesis. On one version of nihilism, the claim is simply that there are no individuals in fundamental reality. Such a view is, of course, compatible with the claim that there are individuals (just not in fundamental reality). In the following, I will leave aside issues of fundamentality, because I think the problem to be discussed confronts all versions of nihilism. See Bacon (2019) for a helpful discussion of fundamentality in distinguishing versions of qualitativism.

2. I am helping myself to thing-talk in setting out the task for the nihilist. The expression 'the way the world is,' for instance, might be ontologically committed to individuals in at least two regards: both ways and the world seem to be individuals, so the demand that the nihilist provide an account of this sort might seem to be unfair. The nihilist will, however, need to come up with her own version of this sort of general demand, so the phrase the way the world is' should be understood as a placeholder for whatever the nihilistically acceptable formulation turns out to be.

3. The question of just what is required for a perspicuous expression of one's view of the world is tricky, but I hope the term is suggestive enough for present purposes. For helpful discussion, see Russell (2018).

4. Hawthorne and Cortens (1995) were the first to develop a sketch of a metaphysics of features. Features are of a different ontological category from individuals: in standard, type-theoretic terms, we may say that unlike individuals, features are not of type $e$. 
corresponding theory in classical, first-order logic. ${ }^{5}$ Recently, Jason Turner 2011) has pressed the objection from notational variance on the basis of a metasemantic principle which, on his view, forces a theory in PF to have the same ontological commitments as one in standard first-order logic. ${ }^{6}$

In this paper, I argue that the metasemantic principle Turner relies on is implausible. Although Turner suggests that the principle requires merely a restricted and unproblematic form of dispositionalism, I contend that it falls prey to the same sort of objections that plague a fully fledged dispositionalist theory. To show this, I develop three cases in which terms obey the supposedly meaning-constraining principle Turner endorses, yet have intuitively different interpretations. These cases illustrate that, pace Turner, individual terms can differ in interpretation without differences in speakers' dispositions to use them. Turner's metasemantic principle therefore fails, and, in consequence, his argument that a theory in PF must have the same ontological commitments as one in FOL is unsound. I then argue that on the nihilist's account, the interpretations of sentences in PF will differ from those of classical predicate logic for reasons similar to those present in the cases I introduce. I thus conclude that Turner's argument for the notational variance thesis poses no barrier to the development of PF-nihilism.

In Section 1, I briefly introduce predicate functorese and explain how the nihilist might use it to translate scientific claims. In Section 2, I present the general problem of notational variance as well as Turner's specific argument against predicate functorese nihilism. Section 3 develops the case against the metasemantic principle Turner employs and Section 4 concludes.

5. In addition to the notational variance objection, PF-nihilism must confront an objection that Andrew Bacon (2019) develops. Bacon argues that PF-nihilism runs afoul of "the insight ... that a property is essentially determined by its behavior on individuals." The trouble is that "the Nihilist wants there to be primitive differences between properties like being an electron and being a proton, even though these properties have exactly the same behavior." As Bacon remarks in a footnote, however, the nihilist can circumvent this objection by using a type theory containing only $t$ and types derived therefrom. Bacon worries that this is not, properly speaking, a form of predicate functor nihilism. In the present context, anything will count as PF-nihilism that employs the logical system developed by Quine et al., regardless of the mapping of syntactic categories to types used. While I will not have space here to develop a suitable type theory, see Diehl (2017) for an account of how this might go.

6 . The notion of notational variance is used in a variety of ways in the literature. For now, we will say that two theories are notational variants iff they differ merely superficially. (See French, in press, for this initial characterization as well as for detailed discussion.) We are thus using the term 'notational variance' for what is also called 'theoretical equivalence' (Woods 2018). Since the use of the terms is not standardized in the literature, those who would prefer to reserve 'notational variance' for a stricter notion may silently replace it with 'theoretical equivalence.' 


\section{Feature-Placing Language}

I shall begin by sketching one way in which an ontological nihilist might express herself informally and then provide a regimentation of this informal language using predicate functorese. On this proposal, the nihilist will use a feature-placing language as an informal language, in place of English or another natural language, and use predicate functorese, in place of classical predicate logic, as her formal language. Ultimately, feature-placing language will serve a second role, however, as a metalanguage for the semantics for predicate functorese. ${ }^{7}$ Turner's argument is directed at the ontological commitments of any theory in predicate functorese, so the view I develop is among his targets.

To develop an informal language suitable for ontological nihilism, we start by considering sentences such as 'It is raining' or 'It is cold' that do not contain subject terms designating objects. ${ }^{8}$ Unlike many of our ordinary sentences that have grammatical subjects designating objects, feature-placing sentences include a 'dummy' subject, 'it,' that does not stand for anything at all. ${ }^{9}$

Feature-placing sentences seem to provide a promising model for the ontological nihilist, because they manage to be truth-apt without their surface grammar requiring ontological commitments to any particular entity. Consider the case of 'It is raining.' As Hawthorne and Cortens remark (1995: 146), the claim that it is raining is not equivalent to an object-committal claim such as the proposition that molecules of $\mathrm{H}_{2} \mathrm{O}$ are falling from the sky, since the claim that it is raining might hold despite the falsity of any particular object-committal claim. While this is not conclusive, it certainly suggests that feature-placing sentences are free of ontological commitments. ${ }^{10}$

As Hawthorne and Cortens suggest, the ontological nihilist should take feature-placing sentences as a guide to what a perspicuous nihilist description of the world would look like. First, instead of saying 'There is a cat,' the nihilist would say 'It is catting' (Hawthorne \& Cortens 1995 148). The nihilist would then add various sorts of adverbial modifications, suitable versions of logical connectives, and non-objectual quantifiers, to enrich the expressive possibilities of the language. The basic picture of the world underlying such a language would be

7. I will not be able to provide more than a sketch of this form of nihilism here.

8. P. F. Strawson was the first to discuss such sentences systematically, calling them 'featureplacing' sentences, because they seem to place or locate a particular feature within the fabric of reality 1959 203).

9. In conversation, it is sometimes suggested that 'it' stands here for 'the world.' This does not affect Turner's critique, however. It is also wholly without support in the linguistics literature.

10. One might worry that the claim nevertheless entails sentences with ontological commitments, such as the sentence 'There is rain.' Pressing this objection requires one to endorse a controversial account of analytic entailment. See Diehl (2017) for details as to why no such entailment is plausible in this case. 
one in which features obtain or fail to do so and in which they stand in complex combinations. ${ }^{11}$

In addition to an informal language, the nihilist needs a formal language to use in place of quantified predicate logic. That is, if, according to Quine in "On What There Is" (1948), a theory is committed to whatever it takes as the values of its bound variables in first-order classical logic, then it seems that the nihilist requires a language that dispenses with quantification. Drawing on work by Schönfinkel, Curry, and others on combinatory logic, Quine develops and refines a type of logic which is equivalent in expressive strength to first-order logic (FOL) (either with or without identity, depending on whether the form of predicate functorese includes an identity relation) but which dispenses with variables in favor of predicates and operators that combine with predicates to form new predicates (see Quine 1960; 1971: 1992). Predicate functorese is a formal language with the structure of our enriched feature-placing language.

The language of predicate functorese, $\mathcal{L}_{P F}$, contains two types of symbols: a stock of nonlogical predicates and six logical functors, which we will write $\rho, \kappa$, $v, l, \bar{l}$, and $\sigma . \kappa$ and $v$ combine zero-place expressions, standing in for 'and' and 'not', respectively; $\rho$ corresponds to the existential quantifier; and the remaining functors change the adicity of relations and alter the order of arguments in a string. ${ }^{12}$

Even though no variables will figure in expressions in PF, it is helpful to introduce the intended interpretation of the functors using a mixed notation, where functors attach to predicates of specified adicity and are followed by variables $x_{1}, x_{2}, \ldots$. This presentation will show how to give an algorithm for 'converting' FOL into PF. ${ }^{13}$ A sentence $\varphi^{*}$ in PF will be the conversion of a sentence $\varphi$ in FOL just in case $\varphi^{*}$ can be produced from $\varphi$ by a series of substitutions according to the following rules:

- $\rho$-derelativization: $\exists x_{n} F x_{1}, \ldots, x_{n-1}, x_{n} \Rightarrow \rho F x_{1}, \ldots, x_{n-1}$.

- $\kappa$-Cartesian multiplication: $F x_{1}, \ldots, x_{m} \wedge G y_{1}, \ldots, y_{n}$ $\Rightarrow \kappa F G x_{1}, \ldots, x_{m}, y_{1}, \ldots, y_{n}$.

11. There are, of course, many subtleties regarding whether such a language is in fact free of ontological commitments. For discussion, see Hawthorne and Cortens (1995), Dasgupta 2009), and Diehl 2017).

12. For details, see Quine (1960) and Burgess (2005). Quine developed several different versions of PF-languages in his later work, and Bacon (1985) and Kuhn (1983) begin from a slightly different basic stock of functors for their axiomatizations and completeness proofs. For a presentation of natural deduction and examples of many proofs in PF, see Bacon (1985).

13. By 'conversion,' I mean a relation looser than strict translation. For instance, it is compatible with 'conversion' that a sentence $\phi$ in FOL will be false whereas the converted sentence, $\phi^{*}$, in PF will be true if, in fact, FOL is not a notational variant of PF. Whether or not $\mathrm{PF}$ turns out to be a notational variant of first-order logic will depend on whether sentences in $\mathrm{PF}$ have the same interpretations as those in FOL. 
- $v$-negation: $\neg F x_{1}, \ldots, x_{n} \Rightarrow v F x_{1}, \ldots, x_{n}$.

- $\bar{\imath}$-major inversion: $F x_{1}, \ldots, x_{n} \Rightarrow \bar{\imath} F x_{2}, \ldots, x_{n}, x_{1}$.

- $\iota$-minor inversion: $F x_{1}, \ldots, x_{n} \Rightarrow \iota F x_{2}, x_{1}, x_{3} \ldots, x_{n}$.

- $\sigma$-reflection: $F x_{1}, \ldots, x_{n}, x_{n} \Rightarrow \sigma F x_{1}, x_{2}, \ldots, x_{n}$.

In the mixed language, the operator $v$ expresses negation in PF and attaches to an $n$-ary predicate to form a new $n$-ary predicate. The operator $\kappa$ combines predicates of adicity $m$ and $n$ to form a complex $m+n$-ary predicate. Major inversion, $\bar{l}$, kicks the initial element of a sequence of variables to the end, while minor inversion, $l$, switches the first two places. In the simple two-place case, the functors are interchangeable: if $L x y$ can informally be read as ' $x$ loves $y$,' then both $\bar{l} L x y$ and $\iota L x y$ can be read as ' $x$ is loved by $y$.' Major and minor inversion suffice to generate any ordering of a sequence of $n$ elements. Reflection, $\sigma$, knocks off a repeated variable in the final place. For instance $L x x$, 'Someone loves himself,' would be converted to $\sigma L x$, which might be read 'Someone self-loves.' Intuitively, $\iota, \bar{l}$, and $\sigma$, do the work of keeping track of variables. Finally, derelativization gets rid of the innermost quantifier, turning an $n$-place predicate with a terminal bound variable into an $n-1$-place predicate. To add identity to PF (which we will call ' $\mathrm{PFI}^{\prime}$ ), we can introduce a two-place predicate, $I$. Like the identity predicate in first-order logic, adding $I$ to the language represents a fundamental increase in expressive power.

The above translation schema gives a procedure for turning any FOL+I sentence into a sentence of PFI. Let's take a very simple example. Since we're interested in how to express quantification in PF, we'll take an example with a single quantifier, $\exists x P x$, where ' $P$ ' will be read ' $\ldots$ is a philosopher.' To translate this into $\mathrm{PF}$, we use the derelativization functor to derive $\rho P$.

Burgess proposes the beginnings of a 'pronunciation guide' to predicate functorese: negation becomes 'doesn't,' major and minor inversion are read 'suffers' or 'undergoes,' Cartesian multiplication becomes 'respectively ... and,' reflection is 'self-,' and derelativization becomes 'just' (2005 100). We begin with 'doth' to signal a complete sentence. Our simple sentence above thus gets pronounced 'Doth just philosophize.' Through grouping devices afforded by our functors, we can come up with a complex predicate that does the tracking work accomplished by variables in FOL. ${ }^{14}$ For a (slightly) more complicated example,

14. The translation scheme is not complete, however: it does not deal with iterations of derelativization.

One suggestion here would be to pronounce $\rho \rho$ as 'pairwise,' $\rho \rho \rho$ as 'triplet-wise,' etc.; so $\rho \rho P$ would be 'Doth pairwise philosophize.' Another option would be to replace each instance of derelativization with 'once,' allowing us to form interspersed negations such as $\rho v \rho P$ as 'Doth once non- and once more philosophize.' Or one might try using numerical adverbs such as 'once,' 'twice,' 'thrice.' 
consider the sentence 'Every integer has a successor.' Using $Z$ for 'integer' and $S$ for 'successor,' we will regiment this in FOL as $\neg \exists x(Z x \wedge \neg \exists y S y x)$. Applying the conversion procedure, we get $v \rho \kappa Z v \rho S$, which, by our pronunciation guide, we will read as 'doth not integer and not just successor.'

Burgess's guide differs in only minor ways from the sort of informal featureplacing language considered previously. In place of 'doth $F$ ' the feature-placing language we considered uses 'it is F-ing,' so our first example would correspond to 'It is just philosophizing' and our second to 'It is not (integering and not just successoring).'

One difficult issue concerns how to give a nihilistically acceptable semantics for predicate functorese. Existing proposals for such a semantics will be unacceptable because they use a domain-theoretic —ultimately set-theoretic-model theory. ${ }^{15}$ To avoid this objection, a suitably enriched feature-placing language might provide a metalanguage for predicate functorese. But giving this is not the task for this paper.

\section{Is Predicate Functorese a Notational Variant of First-Order Logic?}

I have suggested that the ontological nihilist could use predicate functorese to express something akin to 'conversions' of at least the serious scientific and everyday sentences she needs to preserve. ${ }^{16}$ One might, however, suspect that for this very reason predicate functorese is merely a notational variant of first-order logic. Indeed, this seems to be the lesson Quine himself drew from PF in The Ways of Paradox:

When a theory is given the usual quantificational form, the things that the theory accepts as existing are indeed the things that it accepts as the values of its variables of quantification. If a theory is given another form, moreover, there is no sense in asking what the theory accepts as existing except as we are in a position to say how to translate the theory into the usual quantificational form .... When we switch to predicate-functor logic, such a mode of translation is available. (1971: 304) ${ }^{17}$

Thus, according to Quine, a theory in PF will have the same ontological commitments as the corresponding theory stated in the usual quantificational form.

15. At least, they are unacceptable when given a flatfootedly realist construal. See Bacon 1985 and Kuhn (1983) for this presentation.

16. The ontological nihilist does not think that these are meaning-preserving translations of our ordinary sentences. The worry I am about to present, however, is that-contrary to the nihilist's intentions-sentences in PF have the same truth conditions as those of their FOL conversions.

17. For further discussion of Quine's views, see Schaffer 2009. 370). 
This would spell trouble for the nihilist, however, since she wishes to say that the PF versions of our usual object-committing statements provide a perspicuous, ontologically non-committal characterization of reality. ${ }^{18}$ The underlying question here is whether or not there is a 'translation,' in a strict sense, to and from PF.

For two languages to be intertranslatable requires at least that a sentence in one language have the same truth conditions as its translation in the other language. ${ }^{19}$ It is clear that if PF and FOL are intertranslatable in this sense, then any theory in PF must have the same ontological commitments as its translation in FOL, since ontological commitments are a subset of the truth conditions of a theory. ${ }^{20}$

Turner argues that a metasemantic principle that he takes to be highly plausible forces the semantics of PF to agree with those of FOL and thus that the 'conversions' between sentences in PF and those in FOL are translations. Coupled with the thesis that FOL is committed to the existence of the entities that serve as the values of its bound variables, we get the result that PF is ontologically committal.

\subsection{Turner's Argument for Notational Variance}

Turner argues that predicate functorese inherits the ontological commitments of quantificational languages on the basis of a metasemantic principle he calls $\left(^{*}\right)$. Consider two languages, $\mathcal{L}_{1}$ and $\mathcal{L}_{2}$, that differ only in that where $\mathcal{L}_{1}$ uses an expression $\alpha, \mathcal{L}_{2}$ uses an expression $\beta$. If $\varphi_{\alpha}$ is an $\alpha$-containing sentence in $\mathcal{L}_{1}, \varphi_{\beta}$ will be a sentence of $\mathcal{L}_{2}$ just like $\varphi_{\alpha}$ except with $\alpha$ uniformly replaced with $\beta$. The principle then says:

${ }^{*}$ ) If every term (other than $\alpha$ and $\beta$ ) is interpreted in the same way in $\mathcal{L}_{1}$ as it is in $\mathcal{L}_{2}$, and if the speakers of $\mathcal{L}_{1}$ utter $\varphi_{\alpha}$ in all and only the circumstances in which speakers of $\mathcal{L}_{2}$ utter $\varphi_{\beta}$, then $\alpha$ and $\beta$ have the same interpretation also. 2011: 17)

Turner adds two important qualifications. First, "all and only the circumstances" should be interpreted dispositionally: the antecedent of the conditional is not

18. Even if PF and FOL were ontologically equivalent, the symmetry of equivalence would leave open the response that FOL is itself free of ontological commitments, contrary to the standard view. For the original treatment of the symmetry of translation and its application to the problem of reduction, see Alston (1958). Although taking both FOL and PF to be free of ontological commitments is feasible, one then starts to lose one's grip on what would count as an ontologically committal theory at all. I will thus leave this option aside in what follows.

19. This provides only a necessary, not a sufficient, condition for intertranslatability. The details of what more might be required to provide a sufficient condition are controversial. See Woods (2018). For present purposes, nothing will turn on what additional requirements are in play, since I will argue that the sentences of PF on the nihilist's construal will have different truth conditions from those of their FOL-counterparts.

20. See Rayo (2007) for more details concerning the notion of ontological commitment. 
satisfied if the speakers' dispositions to use the term vary in any way. This caveat is introduced to accommodate differences in meaning between a term such as 'green' and one such as 'grue,' where the speakers' usages might match in all actual circumstances, but where they have different dispositions for many possible circumstances (2011) 18). (It is difficult, however, to make a dispositional criterion precise. We will return to this issue later.)

Second, the consequent says that $\alpha$ and $\beta$ are merely intensionally rather than hyperintensionally equivalent (i.e., it would not distinguish predicates such as 'trilateral' and 'triangular' which have the same modal profile but have intuitively different meanings, in a finer-grained sense). ${ }^{21}$

In addition, Turner introduces a caveat regarding the use of the principle: we should not count speakers' dispositions to assert sentences containing both of the terms in question.

... The question is whether, setting aside the way the speakers think these terms interact, we should interpret them the same way; $\left({ }^{*}\right)$ is supposed to give us a guide for determining whether speakers' assertions of this sort are plausible, and as such it should not be overly sensitive to these assertions themselves. (2011: 18-19)

According to Turner, without such a caveat, the principle could not rule that two terms were notational variants that the speakers themselves believed had different meanings. ${ }^{22}$

Turner argues that the $\left(^{*}\right)$ principle can be used to show that predicate functorese must have the same interpretation as FOL-in our terms, they are notational variants-and thus that PF and FOL share the same ontological commitments. Since he assumes that FOL is committed to the entities in the domain of quantification, he thus concludes that PF is also so committed, contrary to the intentions

21. One might wonder why Turner takes mere intensional equivalence to be sufficient for notational variance and suspect that a stronger criterion is required. I think this objection is not relevant to the question of whether a theory in PF differs in its ontological commitments from its FOL-counterpart, because if it does so, then the two theories will differ even extensionally in truth value, viz. some statement in FOL will be true only if there are objects in the world, whereas the corresponding PF-statement will be true in non-objectual worlds as well. There is an additional question of whether the nihilist's statements in PF are incompatible with the existence of objects or merely not committed to them. I will not settle this question here, as it is not required to establish a difference in possible truth value. The nihilist holds that her PF-sentences have different truth conditions from those of a corresponding theory in FOL; the objector holds that, contrary to the nihilist's claims, PF-statements are forced by $\left({ }^{*}\right)$ to be truth-conditionally equivalent.

22. There is a difficulty here: since the first community will use $\phi_{\alpha}$ in exactly those circumstances in which the second community uses $\phi_{\beta}$ but the languages will be otherwise identical, neither community will be disposed to utter any sentences that contain both the terms. Perhaps this caveat should instead be understood to bar sentences either mentioning both terms or using one term and mentioning the other. 
of the nihilist. Turner stipulates that there could be several intermediate languages between PF and FOL and then uses the $\left(^{*}\right)$ principle to argue that these languages are notational variants of one another and then that they possess the same ontological commitments as PF and FOL. ${ }^{23}$ These intermediate languages differ only in a single symbol, so $\left(^{*}\right)$ can be applied to them.

In order to construct these languages, Turner draws a distinction between two functions played by the quantifier in FOL: the variable-binding role, which Turner claims is ontologically innocent, and the 'There is' role, which does the work of proper existential commitment 2011: 13-15). Let us grant him this distinction for present purposes.

Based on this reflection, he asks us to consider a language, $\mathrm{FOL}^{*}$, that separates these two functions by introducing $\lambda$-s to bind variables so that an open expression, $\varphi$, becomes $\lambda x \varphi$, which is to be read as ' $\ldots$ is an $x$ such that $\varphi^{\prime}$. FOL ${ }^{*}$ possesses a single sentential operator, $\bar{o}$, which bears existential commitment. ${ }^{24} \mathrm{Next}$, we introduce a language, $\mathrm{PF}^{*}$, that is just like $\mathrm{FOL}^{*}$, except that instead of the operator $\bar{o}$, this language uses the derelativization functor, $\rho$.

Here, then, is my reconstruction of Turner's argument: 25

1. $(*)$

2. $\mathrm{FOL}^{*}$ speakers have the same dispositions to assert $\varphi_{\bar{o}}$ as $\mathrm{PF}^{*}$-speakers have to assert $\varphi_{\rho}$.

3. By (1) and (2), $\bar{o}$ and $\rho$ have the same interpretation.

4. By (3), $\mathrm{FOL}^{*}$ and $\mathrm{PF}^{*}$ are ontologically equivalent, since $\mathrm{FOL}^{*}$ and $\mathrm{PF}^{*}$ differ only by $\bar{o}$ and $\rho$.

5. By construction, FOL and $\mathrm{FOL}^{*}$ are ontologically equivalent.

6. By construction, $\mathrm{PF}$ and $\mathrm{PF}^{*}$ are ontologically equivalent.

7. By (4)-(6), PF and FOL are ontologically equivalent.

\section{Objections to Turner's Argument against Functorese Nihilism}

Although Turner's argument against PF-nihilism depends upon the $\left(^{*}\right)$-principle, he provides little in the way of defense for it. He motivates the principle by asking

23. Strictly speaking, theories stated in languages, rather than languages themselves, possess ontological commitments. Speaking loosely, however, we may say that two languages have the same ontological commitments just in case corresponding theories stated in the two languages have the same ontological commitments. This criterion is merely heuristic, however, and we will make this premise explicit in the statement of Turner's argument.

24. I have altered Turner's notation slightly for readability.

25. Cf. also Turner's (2016) recent reiteration of this argument. 
the reader to imagine encountering a man who-puzzlingly and mysteriouslyinsists on saying 'eulb' on just those occasions we would say 'blue' without providing any account of his usage. If we were to observe his behavior long enough, Turner says, we should conclude that his 'eulb' has the same interpretation as 'blue' (2011: 13-14).

Turner is surely correct that this would be a natural interpretative strategy, but it falls far short of a defense of $\left(^{*}\right)$. $\left(^{*}\right)$ is a principle governing what the possible interpretations of a term are, not of how we should interpret a speaker who confuses us. It might be a good guide to radical translation, but it only serves as a good guide to meaning if we take the controversial—and, I think, ultimately unsustainable-view that interpretation under radical translation is equivalent to meaning. $\left({ }^{*}\right)$, after all, is supposed to be a metasemantic principle that constrains any possible semantics for a term. In my view, this encodes two substantive and controversial commitments regarding metasemantics that an ontological nihilist has no reason to accept.

First, Turner assumes that there could not be a further fact of the matter concerning the contributions of the term to truth conditions beyond those principles that a community should follow in interpreting a deviant speaker's use of a term. Second, $\left(^{*}\right)$ is based on a dispositional criterion of meaning that requires the truth conditions of a term to supervene on the circumstances in which its speakers would use this term. While dispositional criteria do have defenders, they are highly controversial in light of Kripke's skeptical arguments. ${ }^{26}$ In particular, such criteria do not allow for principled ways of distinguishing between error and differences in meanings.

I shall argue that Turner's dispositional principle $\left(^{*}\right)$ falls prey to several familiar problems attending dispositional criteria in general. Now, I do not hope to convince the dedicated dispositionalist. Instead, I seek to show that $\left(^{*}\right)$ commits Turner to full dispositionalism and thus that it is question-begging for Turner to invoke such a principle in this context. He provides little in the way of guidance regarding the proper use of this principle, and I will argue that there is no way to make the criterion precise that respects our intuitive judgments concerning differences in meaning between terms. I will consider three cases in which Turner's criterion delivers the wrong result concerning the meanings of terms. Finally, I will draw some general lessons concerning why the principle cannot be modified to press the notational variance objection without begging the question.

26. See Kripke (1982). For defenses of dispositionalism against Kripke's skeptical argument, see Fodor (1990) and Blackburn (1984). See also Boghossian (1989) and Hattiangadi (2007) for valuable discussion. In my view, the defenses of dispositionalism do not address the underlying challenge posed by Kripke, which I shall develop in the counterexamples below. Unfortunately I cannot defend this fully in the present context, but I will provide several hints of why I think this is at specific points in what follows. 
The general problem regarding the criterion-and the counterexamples I will suggest develop this point-comes from an unclarity surrounding the very notion of a disposition. Intuitively, there's some sort of connection between having a disposition and satisfying certain counterfactuals, but it is notoriously difficult to specify which counterfactuals these are. ${ }^{27}$ In particular, do we have to consider how speakers would react if they were to become aware of the facts determining the reference of their terms? On one common picture, these would be facts involving the specific causal chains from particular objects to speakers' original utterances of terms. For instance, the reference of the term 'water' would be determined by water's standing in a certain place in a chain leading to the original speaker's utterance of 'water.'

The question whether we have to consider speakers' possible reactions upon becoming aware of such facts leads to a dilemma for the dispositional theorist. On the one hand, in certain cases, the difference in the meaning of terms cannot be gleaned from speakers' dispositions without considering how these speakers would react when made aware of the reference-fixing facts. The first case we will consider involves inversions in color dispositions. If we do not consider the reference-fixing facts-in this case, the speakers' qualia-Turner's principle will incorrectly characterize the inverted uses of terms as equivalent.

On the other hand, including reactions when learning reference-fixing facts would render the principle diagnostically ineffective, since to judge if two terms differed in meaning, we would have to know the reference of these terms. In the spectrum inversion case, once we have stipulated that the reference-fixing facts include different patterns of qualia, we thereby know that the two communities' terms differ in meaning and there is no need for further inquiry. Correspondingly, ignorance about whether two communities' terms are equivalent in meaning entails that we do not know whether the underlying reference-fixing facts differ. We shall thus see that in the case of Turner's principle, there is no way to get a class of counterfactuals that will both respect intuitive differences in the meanings of terms and can be applied in a non-question-begging way.

\subsection{Inverted Colors}

The first sort of case we will consider concerns situations in which two communities' use of terms with different meanings is disguised by inversions in the

27. Fodor 1990 pushes back against Kripke's claim that uncertainty about which counterfactuals obtain renders dispositional criteria unworkable. Fodor notes that we do not know many counterfactuals in the case of physical laws, but this does not and should not lead us to question the determinacy of these laws. Fodor's response, however, does not address the particular type of dilemma I propose for the dispositionalist. 
community members' dispositional profiles. ${ }^{28}$ The initial thought behind the case comes from instances of what I will call 'symmetrical terms', where terms $\alpha$ and $\beta$ are symmetrical iff a community of $\mathcal{L}_{1}$-speakers uses $\alpha$ to mean $m_{1}$ and $\beta$ to mean $m_{2}$ and a community of $\mathcal{L}_{2}$-speakers uses the terms with switched meanings. If $\mathcal{L}_{1}$-speakers have inverted dispositional patterns, however, the differences in meanings between the terms in these different communities will be masked. This simple description of the case does not quite get us to a counterexample to Turner's principle, because differences between the languages will concern a pair of terms. The basic idea can be preserved, however, in a slightly more complicated form.

According to one metaphysics of color, color terms refer to relational properties of objects to cause a particular type of quale in an observer in world $w$ given the physiology of the observer in $w$. 'Red' refers to the property of being such as to cause the red quale in perceivers in world $w$ with the physiology that these beings have in $w$, 'green' to the property of being such as to cause the green quale in perceivers in $w$ with the physiology that they have in $w$, and so on. ${ }^{29}$

Consider two worlds, $w_{1}$ and $w_{2}$. In $w_{1}$, there are speaking beings who have the red quale when we would have the green quale as well as when we would have the red quale. ${ }^{30}$ Members of community $C_{1}$ speak a variety of English: they make all the same sort of assertions we do except that, systematically, they say 'red' both when we would say 'red' and when we would say 'green.' In $w_{2}$, imagine a community, $C_{2}$, of beings that have the green quale both when we would have the green quale and when we would have the red quale. Members of this community use 'green' in just those situations in which members of the other community say 'red,' viz. when they experience things which would cause us to say either 'green' or 'red.' In both languages, color terms are simple terms.

The two communities would seem to be disposed to use 'green' and 'red' in the same circumstances, since the difference between the meanings of the two terms will be compensated for by the inverted qualia profiles of the speakers. By $\left.{ }^{*}\right)$, we would thus have to conclude that 'red' in the first community's language

28. Turner's principle, as noted above, is supposed to hold for interpretations-coarsegrained meanings-rather than fine-grained meanings. The sort of meaning differences I will discuss in the following three cases are all differences in the truth conditions for the terms and thus do not require any merely hyperintensional differences.

29. Readers may find the suggested account of the metaphysics of color implausible and suspect that both color terms and color qualia should be indexed to worlds. Furthermore, one might worry that red and green qualia are not relevantly symmetric and thus that the inverted scenario is not metaphysically possible. This suspicion may well be justified, but this is irrelevant for current purposes. Nothing hangs on color terms in fact working in the manner suggested. It is enough that the meaning of some pair of terms could be determined in this way for Turner's general criterion to fail. This seems to be obviously the case.

30. They do not have to be beings of the same natural kind as we are. All we need is for them to speak a language like ours and to have relevantly similar kinds of qualia. 
has the same interpretation as 'green' in the other community's. By stipulation, however, beings in the first community say 'red' when they are having red qualia and beings in the second say 'green' when having green qualia, so 'red' and 'green' pick out different relational properties: '... is red' in the first language means $<$ is such as to produce the red quale in $C_{1}$ beings $>$ and ' $\ldots$ is green' in the second means $<$ is such as to produce the green quale in $C_{2}$ beings $>$.

While it is clear that 'red' and 'green' denote hyperintensionally distinct properties, one might suspect that they are intensionally equivalent: after all, at least in nomologically similar worlds, the very same things that produce the red quale in the members of $C_{1}$ produce the green quale in members of $C_{2} \cdot{ }^{31}$ As noted, the $(*)$ principle maintains only that two dispositionally equivalent terms are intensionally equivalent, so for our case to constitute a counterexample, 'red' and 'green' would have to differ intensionally. Examination of slightly more complex cases shows, however, that 'red' and 'green' make a truth-functional difference when qualia themselves, rather than the objects producing the qualia, are at issue, and are therefore intensionally distinct.

Consider the open sentence '... is the kind of quale produced by red things', as used by members of the $C_{1}$ community. Since 'red' is part of $C_{1}$ 's language and $C_{1}$ speakers have the red quale when they see things they describe using the word 'red' (i.e., both red and green things), this will apply to red qualia. But the open sentence '.. is the kind of quale produced by green things', as used by members of the $C_{2}$ community, will not apply to red qualia, because speakers of $C_{2}$ do not have red qualia when they see things they describe using the word 'green' (i.e., both red and green things); instead, they have green qualia. Thus 'red' and 'green' can make a truth-functional difference and are intensionally distinct. Nevertheless, this is a difference that does not show up in different dispositional patterns, because the very same circumstances will lead each community to say the corresponding utterance: facts about differences in the phenomenal character of qualia are not the kind of facts that can be manifested in differences in usage in a public language, for speakers have no way to pick out the nature of their qualia directly except by reference to the objects that cause them.

One might try to block this case by including among the counterfactuals relevant to the speakers' dispositional profile counterfactuals concerning what would occur if the speakers had inverted qualia. If we asked what would happen if the first community had the second community's qualia profile, then there would be a difference between the two communities' dispositions to use 'red' and 'green': in the context in which the first community had the second community's qualia profile, the first community would use 'red' differently from how the second uses 'green.' Is it legitimate, however, to assume that the meaning of these terms would remain the same if the qualia of the speakers were different? When

31. My thanks to an anonymous reviewer for raising this worry.

Ergo • vol. 5, no. $37 \cdot 2018$ 
we look at standard cases, it seems as though it is not. ${ }^{32}$

First, it strains credulity that each community would use the terms without meaning change if their qualia were relevantly different. If we try to imagine a case in which, say, the 'green'-community-all of whose members had grown up with just one sort of qualia-suddenly acquired qualia of another kind, then it seems that their whole speech patterns (and thus also the meaning of 'green') would be thrown up in the air. It likewise appears as if changing the physiology of the red community is going to destroy the relevant linguistic practice. Thus, expanding our consideration of the use patterns to include these worlds will not break the symmetry between these communities' uses of 'red' and 'green.'

This raises the second worry: the only reason to introduce the symmetrybreaking distinctions Turner requires is to assume, question-beggingly, that the meanings are as we intuitively judge them to be. In order to save the dispositional view, Turner needs the characterization of the worlds under consideration to be determined by our intuitions of meanings, rather than having the dispositions do the explanatory work. ${ }^{33}$ In the qualia case, if we were to build into the example that the first community would differ in their dispositions if their qualia profiles

32. For the canonical articulation of this strategy, see Kripke's discussion of whether a dispositional criterion can help us distinguish plus from quus, where the extension of quus differs from that of plus only for large, as yet uncalculated, inputs 1982 22-37). The trouble, Kripke says, is that we can modify our example slightly so that addition and quaddition differ only in their answers to some calculation that exceeds actual human subjects' finite computational power 1982 26-27). Blackburn (1984) pushes back against this point, arguing that our notational method for the addition of large numbers by summing each column and then carrying provides a suitable algorithm for the computation of arbitrarily large numbers. Blackburn's idea here is that the idealization we need to perform to rule out quaddition is harmless because we are imagining the extension of a single 'track' of cognitive capabilities, rather than speculating about a complex multi-track disposition. Whether or not Blackburn's response to Kripke's original case is compelling, it will not succeed in the case of inverted qualia, because the changes in physiology of the beings would create a qualitatively different situation that cannot reasonably be considered an extension of previous tendencies.

Can we, Kripke then asks, expand our dispositional criterion to include what we would do were our computational powers greater than they are? He offers two responses, both of which are relevant to Turner's use of the dispositional principle. These two responses are usually summarized as the problem of infinity and the problem of distinguishing mistaken uses from correct uses of a term with a different meaning. I am dividing up the landscape in a slightly different way, because it is more helpful for considering the specific case at hand.

33. This second problem demonstrates why Fodor's (1990) response is ineffectual. Fodor claims that our ignorance regarding specific counterfactuals is typically compatible with our knowledge of general laws. He cites the case of Newton's laws of motion as an example. For instance, we cannot, he claims, say what would be the case if there were no friction. Even if Fodor is right about this, however, the response is dialectically inappropriate in the current context. In the case Fodor sketches, we are supposed to have independent access to the laws, whereas in the present case, the idea is to use the dispositional facts to derive the meaning facts. In the latter sort of context, our in-principle ignorance of what would be true about dispositional meaning facts becomes relevant. 
were switched, then this would similarly deliver the desired difference of meaning but would require that we hand-write into the evaluation of the dispositional criterion itself a result that is based entirely on our foregoing judgment that 'red' and 'green' do differ in meaning as uttered by the two communities. ${ }^{34}$ If it is our pre-theoretical intuition that is driving our evaluation of the speakers' dispositions, then the criterion is otiose.

Now, the advocate for Turner's criterion might object here that we have stipulated the case in a way that prejudges matters against the dispositionalist by building in a difference of meaning that floats free of dispositional patterns of use. In the present context, however, this response gets the dialectic wrong: all that is required to oppose Turner's principle is that there can be an intuitive difference in meaning despite the sameness of dispositional profiles. The case of inverted qualia seems to provide one such instance.

\subsection{VN-Numbers and Z-Numbers}

For a second sort of case in which Turner's principle fails to predict an intuitive difference in meaning, consider a modification of Paul Benacerraf's story involving the two characters Ernie and Johnny (Benacerraf 1965 55). For the purpose of our example, assume that Platonism is true. In particular, let us adopt the candidate metaphysical view that no cardinal number is identical to any set, although ordered collections of sets can be isomorphic to the cardinal numbers. In our version, Ernie and Johnny learn arithmetic and set theory at the same time. At some point, Ernie grows curious about the relationship between the natural numbers and the set-theoretical entities he's been studying. His teacher, Marnie, has always had a predilection for the von Neumann ordinals and so she tells him that although the natural numbers are ontologically distinct from the finite von Neumann ordinals, the von Neumann ordinals have a special relationship with the natural numbers. To mark this special relationship, she teaches him to use the term 'VN-number' to refer to the finite von Neumann ordinals, each of which is associated with a corresponding natural number. She shows him that some general arithmetical relationships can be expressed using 'VN-number.' For instance, Ernie can state that there are infinitely many VN-numbers. Furthermore, she tells him that every statement about natural numbers has a corresponding statement about VN-numbers, and, in order to highlight the close relationship, he begins to use 'VN-number' in arithmetical statements where he would have previously used 'natural number.' Johnny's teacher, however, prefers the Zermelo

34. In the case of addition and quaddition, if we were to require an answer as to what the speaker would do for every possible case, then we would be able to distinguish between addition and quaddition but determining the speaker's dispositions would require that we already have the full story regarding her intentions-which was what we wanted to find out in the first place (Kripke 1982 28). 
sequence, so she tells Johnny to use the term 'Z-number' to refer to a Zermelo ordinal, and the rest of the story follows in a parallel fashion.

Now, imagine that they forget all the set theory they know. They both subsequently just use the language of arithmetic and the additional predicate ' $\mathrm{VN}$ number' or 'Z-number,' but their languages differ in that Ernie says 'VN-number' where Johnny says 'Z-number.' According to $\left({ }^{*}\right)$, 'VN-number' and 'Z-number' have the same meaning because Ernie and Johnny will use them in all the same possible contexts, since they will agree on everything statable within the language of arithmetic. On the other hand, on the assumption that the meaning of a term depends on the act of reference fixing, 'VN-number' will be interpreted differently from 'Z-number' because the extension of 'VN-number' is the set of finite von Neumann ordinals. (Similar reasoning will hold for the predicate 'Z-number.')

One might reply on behalf of Turner here that Ernie and Johnny do differ in their dispositions to use 'VN-number' and 'Z-number': if they were re-taught set theory and learned the facts about how the reference of their terms was fixed, then they would no longer use them in the same circumstances. ${ }^{35}$ As in the previous case, however, this would render the $\left(^{*}\right)$ principle toothless: what would be doing the work here would be the pre-theoretic intuition of a difference in meaning, rather than the dispositions themselves, as required for Turner's case.

This can't be the way Turner intends the principle to be used. If it were, then whenever the meaning of terms differed, dispositions to use the terms would likewise differ since we would have to consider how we would use the terms if we became aware of what the meanings in fact were. If this were the correct interpretation of the principle, then it would be question-begging ever to assume that the principle applied to a specific case, since it would build in that there was no difference in the meaning of the terms-since otherwise the communities would use the terms differently when finding out about this difference.

\subsection{The GD-Community}

Finally, we will examine a case that has several important similarities with that of functorese nihilism. Consider a very religious community that doesn't think of things as merely existing but as present by a decree of God. The idea here is that when, for instance, this community encounters a table, they take the table's

35. This is not the only thing they might learn that would cause them to be disposed to use the terms differently: they might also learn that 'VN-number' expresses the purportedly natural correlation between arithmetical facts and facts about von Neumann ordinals, and 'Z-number' expresses the corresponding facts about Zermelo ordinals. In this case, Ernie and Johnny would be learning semantic facts directly rather than metasemantic ones concerning how the references of their terms was fixed. I focus on the metasemantic case, however, because I take it that it is clear that adding direct semantic knowledge would render Turner's criterion unworkable. 
presence as the manifestation of a divine decree. They use a syntactically simple operator $\mathrm{G}$ to express the thought that we would express using the syntactically complex 'God decrees that there is ...' They use this operator in just the same circumstances in which we would assert existence, because whenever we see bare existence, they attribute divine intention.

Now, this community has a language, similar to FOL, that it uses to express its scientific claims. It then uses a $\lambda$-expression for variable binding. Such a language would differ from $\mathrm{FOL}^{*}$ as spoken by atheist scientists only by using $\mathrm{G}$ where the $\mathrm{FOL}^{*}$ speakers would use $\bar{o}$, the existential force-carrying operator in $\mathrm{FOL}^{*}$, which we shall pronounce in English as 'there is.' Now, within the G-language, the community's use of the G operator would match that of FOL*'s ontological commitment. According to $(*), G$ should have the same meaning as $\bar{o}$. Now it might seem as though the difference between $G$ and $\bar{o}$ could be merely hyperintensional, since we have stipulated that they would use $G$ in the same possible circumstances as the atheistic community used $\bar{o}$. This impression is incorrect, however: their assertions differ truth-conditionally from those of the atheistic community. If, that is, it is not the case that God decrees that there is a table, the GD-community's claim will be false even when the non-religious community's statement is true. Thus, the intuitive difference between the two languages is in the interpretation of the terms, not more fine-grained meanings.

If it is possible for there to be a community with the intentions I have described, then intentions-as specified in a metalanguage inaccessible to the speakers-can vary between communities even when a translation principle is specified and the two communities differ in their use only of the term in question. Perhaps, however, one might doubt that the religious community could really intend something syntactically complex-something, that is, with the content we would express with 'God decrees that there is ...'-using a syntactically simple single operator. ${ }^{36}$ This objection relies, however, on assuming a perfect correlation between the syntax of a language and the complexity of its contents. But this assumption is clearly belied by the example of English, where we use syntactically simple symbols for complex contents all the time. It is only after long theoretical investigation that the complexity of the content is revealed. Consider, for example, the case of the quantifier itself. If Turner is right, the existential quantifier in English performs two very different jobs: that of variable binding and that of existence assertion. Its syntactic simplicity in languages with which we are familiar hides the complexity of its contents. The idea behind the GD-community is that their G works in a similar way: it is just that rather than the two functions of the quantifier, they envision a complex divine act granting existence.

Second, one might worry that the scenario only appears to be possible because of the brevity of our sketch, and that once we look at how the translations work

36. This objection is due to an anonymous referee. 
in practice, we will no longer be able to maintain that there really is a difference in intention. This worry comes to the fore when we examine the behavior of our new operator under negation. Here, one might suspect that once we have forced G-negation to operate like classical negation, we will no longer be able to make out a difference in meaning between the religious community and our own. ${ }^{37}$ This objection is important, so I will lay out the problem in more detail and then explain why I think the envisioned scenario is nevertheless possible.

FOL $^{*}$ speakers who adhere to classical logic accept the following two schematic formulas for existential generalization (where ' $a$ ' is a schematic letter for an individual):

(1) $F a \rightarrow \bar{o} \lambda x$. $(F x)$

(2) $\neg F a \rightarrow \bar{o} \lambda x$. $(\neg F x)$

By stipulation, the G-community will accept all the corresponding formulas as our $\mathrm{FOL}^{*}$-community, in particular:

(3) $\mathrm{Fa} \rightarrow \mathrm{G} \lambda x$.(Fx)

(4) $\neg F a \rightarrow G \lambda x$. $(\neg F x)$

These schemas ensure that negations will scope correctly in both the ordinary and in the G-communities. Together with the schema of the law of excluded middle, common to both languages,

(5) $F a \vee \neg F a$

these principles allow for intuitive translations of natural language statements with 'every.' Consider the case of 'Every electron orbits a proton.' The FOL*community will write

(6) $\neg \bar{o} \lambda x$. (Electron $x \wedge \neg \bar{o} \lambda y$. (Proton $y \wedge x$ orbits $y))$.

The equivalent $\mathrm{G}$ sentence will be:

(7) $\neg \mathrm{G} \lambda x$. (Electron $x \wedge \neg G \lambda y$. (Proton $y \wedge x$ orbits $y))$.

But this seems to express the English sentence (adding variables for readability) 'It is not the case that God decrees that there is an electron $x$ such that it is not the case that God decrees that there is a proton $y$ and $x$ orbits $y^{\prime}$ and one might worry that God has simply failed to make a decree in this instance.

Notice, however, that even though one could imagine a sort of GD-community that would not insist that God makes a decree on every matter and that this community's pattern of use would not satisfy the antecedent of $(*)$, we can also

37. Thanks to an anonymous referee for suggesting this worry. 
envision a community whose God was omni-opinionated. Let this latter community be our original GD-folk. They will think that God makes a decree in every case-and thus will preserve the correspondence between the two communities' assertions. This by no means prevents there being a difference in meaning between the two cases as long as they are in fact saying that God is making it be the case or making it fail to be the case. There does not seem to be anything incoherent about such a stipulation, and thus no reason to think that the correspondence in behavior under negation will force the two meanings to be equivalent.

Now, what lessons can we draw from this for Turner's $\left({ }^{*}\right)$ principle and his arguments concerning predicate functorese nihilism more generally? If we can imagine that there are two communities that have radically different ideologies while having the same dispositions to accept the relevant symbols, then we have an additional counterexample to Turner's principle. But one might doubt that these two communities have all the same dispositions-after all, isn't it the case that with more expressive resources, they would disagree about further sentences? According to this objection, we have forced the interpretation of the sentences to come apart by interpreting them in a common metalanguage, English, but if the speakers of the language were to come to learn this language, then there would be additional differences in their linguistic behavior. Now, once again, the Kripkean doubts introduced in our discussion of the color case should make us wary of understanding the dispositional criterion so as to include what speakers would do in idealized epistemic situations, but there is another problem with the proposed extension in the current case.

Let us now imagine that both the FOL* speakers and the G-speakers have additional linguistic resources to express certain ontological, theological, and metalinguistic claims-for instance, to talk about the meaning of their G-utterances. Now, it might seem that if we consider these additional resources as part of the languages of the two communities, then the antecedent of $\left(^{*}\right)$ will no longer be satisfied. But remember that Turner bars certain kinds of statements from consideration in determining the interpretation of an individual term. In particular, he bars metalinguistic statements and statements in 'mixed languages'-languages that use both resources. But these are exactly the sort of resources that are required to generate sentences that our two candidate communities will disagree about. So even if we allow that these two communities will have dispositions to accept different sentences, the antecedent of $\left({ }^{*}\right)$ will be satisfied and we will have a counterexample.

\subsection{The Background Theory Denied by the Nihilist}

The GD-community's statements intuitively differ in interpretation from those of FOL-speakers, because the GD-community has a background theory-a theologi- 
cal doctrine-not possessed by the FOL-speaking community. ${ }^{38}$ In this section, I will claim that the functorese nihilist and the object-committed ontologist are in an analogous situation. I suggest that the truth of existentially committal statements of FOL (such as $\exists x C x$ ) requires more than that a pattern of features obtains. In particular, it requires that there be a difference in kind between things and what is possessed by those things (properties). ${ }^{39}$ On this story, for there to be things in the sense asserted by existentially quantified statements requires that there be both identity and distinctness facts concerning entities and for there to be entities in which features inhere. According to the PF-nihilist, however, the corresponding PF-statements do not require that there be entities in which features inhere but simply that there be a pattern of features. In other words, they deny the existence of two types of entities-individuals and properties-with the latter inhering in the former.

We can examine the outlines of this strategy by altering our description of the GD and $\mathrm{FOL}^{*}$ communities' languages so that the communities themselves could articulate their background commitments. Imagine that the GD and FOL* communities have a wide range of linguistic resources at their disposal, which they use to articulate their theological commitments. Nevertheless, all of their scientific claims are made within the more restricted languages, $\mathrm{FOL}^{*}$ and the Glanguage described above. Since the statements of the fragments of the language are interpreted relative to the broader languages, it should be uncontroversial that the statements of the two fragments differ in meaning, despite the existence of a translation (in the weak sense) from the statements of the atheist community to those of the theological community.

A parallel account is available to the ontological nihilist who uses predicate functorese: she can avail herself of additional linguistic resources to distinguish her view from that of the non-nihilist. This sort of nihilist will satisfy Turner's demand that she provide (rough) translations of ordinary and scientific statements, but these statements will be interpreted in a nihilistically acceptable way using additional vocabulary. She will say that ordinary and scientific statements are strictly speaking false because they are committed to a background theory on which there are things in which particular properties inhere, but that there are statements in PF that capture nearby true claims. According to such a nihilist, there are substantive ontological requirements for quantificational claims to be true. In particular, there must be things that satisfy two theoretical roles: the individuator role and the bearer-of-properties role. The first consists in there

38. My thanks to an anonymous referee for suggesting this way of framing the issue.

39. I will help myself here to property-talk, but I think the fundamental picture is one that can be reformulated using type-theory in a manner acceptable to the property nominalist. In brief, it requires only that there be a difference in kind between entities of type $e$ and those of all other types. 
being facts regarding the identity and distinctness of entities. The second consists in there being something in which properties inhere but which itself does not inhere in anything: in type-theoretic terms, type $e$ entities are those to which entities of other types can be applied but which cannot be applied to any other type. Something like these roles is codified in Aristotelian accounts of substance, but they are reflective of folk ontological commitments to a difference in nature between things and the way that these things are. The nihilist, however, claims that the traditional picture is incorrect. ${ }^{40}$ Thus the nihilist claims that 'There is a cat,' which is correctly symbolized by $\exists x C x$, is literally false since its truth would require an object ontology. Nevertheless, it conveys information better captured by the true $\rho C$.

The PF-nihilist should treat philosophical claims articulating the theory of ontology-presumably phrased in either a higher-order language or one with additional vocabulary-as false as well. Unlike ordinary and scientific statements, however, they do not have true statements in the vicinity, according to the nihilist. Here the key claim will be something like: 'the bearer role and the individuator role are fulfilled.' The nihilist will not translate the specifically ontological language used in this statement into feature-placing terms, because this language describes the characteristic ideology of object-committal ontology. If we count statements in this expanded language, then we will have a difference in usepatterns. Thus, in a sense, this provides an additional response to someone who remains attracted by the $\left(^{*}\right)$ principle. The functorese nihilist will have different dispositions from those of the standard ontologist, because she will differ with regard to affirmations or denials of the statements of ontology.

Turner might respond here that we have counted differences in use-patterns in contexts where both of the terms are used and that this is insufficient to establish differences in meaning. But consider whether such a response is plausible: we have an entire realm of theorizing in which the functorese nihilist will disagree with the standard ontologist. Why should we discount everything the functorese nihilist says in the metalanguage about ontology? Why should we accord absolutely no consideration to her usage patterns with regard to all ontological questions? If we allow such differences to count, then Turner's description of the functorese nihilist will not obtain.

At this point, we might wonder why the nihilist requires predicate functorese at all..$^{4}$ Why shouldn't the nihilist simply use an FOL-like language as her language of choice, but disavow the ontological theory that she claims is presupposed

40. See Diehl (2017) and Diehl 2018) for an exposition and defense of this view as an account of the commitments of ordinary and scientific statements. Thanks to an anonymous referee for pressing me to clarify the metaontological background story here.

41. Thanks to an anonymous referee for raising this concern and for helpful suggestions regarding the dialectic. 
by ordinary FOL assertions? In other words, while she would claim that ordinary uses of FOL presuppose a heavyweight theory of things on which the two roles of things are fulfilled, couldn't she introduce a lightweight quantifier that would operate in a structurally analogous way without being committed to this ontology? This strategy is indeed possible: there is nothing about the shape of the symbols in FOL or PF that incurs ontological commitment. Nevertheless, even though a nihilist could introduce such an FOL-light language, there would be disadvantages to using such a language rather than PF. An FOL-light language would be less ontologically perspicuous than PF, because FOL-light includes notation-the quantifier and variable structure-that does not correspond to anything in reality. $\mathrm{PF}$, by contrast, does not include this sort of superfluous structure. Thus, even if FOL-light could be implemented, the nihilist is motivated to turn to PF as her language of choice.

\section{Conclusion}

I have argued (1) that Turner's metasemantic principle $(*)$ should be rejected and (2) that the PF-nihilist does not have to accept sentences corresponding to all of those of the standard ontologist. If these reasons are correct, then his argument for the conclusion that a theory stated in PF inherits the ontological commitments of its FOL-counterpart fails. If we use a feature-placing metalanguage that is itself nihilistically acceptable, then PF provides a promising basis for a nihilistically suitable language.

Much positive work remains in developing PF-nihilism. Here are a few initial questions: how can the nihilist accommodate modal operators and second-order quantifiers? How are intentional attitudes to be represented? And how is identity to be understood in a nihilistically friendly context? Finally, is PF speakable as a first language and, if so, by beings of what sort? If it is not, how can the nihilist assure us that we understand her position and are not simply piggy-backing off our understanding of ontologically committal language?

But I see many reasons to be hopeful here: Hawthorne and Cortens give a first indication of how a limited resource of English, feature-placing constructions, might be generalized. Insofar as we understand feature-placing constructions in natural language, there seems to be no obstacle to expanding their number. I have also suggested a way in which a non-object committing semantics for PF might be developed. Finally, it would seem that PF has all the hallmarks of a speakable language: it has a clear compositional structure in accordance with a few simple rules. While its combinatory principles operate differently from those of familiar natural languages, this should not create any deep difficulty in learning it. Thus, the prospects for using PF as the nihilist's fundamental language appear good. There is much work to be done on the language, logic, 
and metaphysics of nihilism, but it seems that functorese nihilism can meet the expressibility challenge.

\section{Acknowledgements}

My thanks to Benj Hellie, Nora Kreft, Michael Miller, Richard Moore, Nick Stang, Tobias Rosefeldt, Thomas Sattig, Andrew Stephenson, Barbara Vetter, Lisa Vogt, Jessica Wilson, Jack Woods, and Julia Zakkou, as well as the members of the PERSP research group in Barcelona and Mari Mikkola's colloquium at the Humboldt University in Berlin for valuable discussion, suggestion, and critique at various stages of this project. Special thanks to Beau Madison Mount, without whom this paper would not exist. His insight and ideas have shaped this paper throughout.

\section{References}

Alston, William (1958). Ontological Commitments. Philosophical Studies, 9(1-2), $8-17$.

Bacon, Andrew (2019). Is Reality Fundamentally Qualitative? Philosophical Studies, 176(1), 259-295.

Bacon, John (1985). The Completeness of a Predicate-Functor Logic. Journal of Philosophical Logic, 50(4), 903-926.

Benacerraf, Paul (1965). What Numbers Could Not Be. Philosophical Review, 74(1), 47-73.

Blackburn, Simon (1984). The Individual Strikes Back. Synthese, 58(3), 281-301.

Boghossian, Paul (1989). The Rule-Following Considerations, Mind, 98(392), $507-49$.

Boolos, George (1984). To Be is to Be the Value of a Variable (or to Be Some Values of Some Variables). Journal of Philosophy, 81(8), 430-449.

Burgess, John P. (2005). Being Explained Away. Harvard Review of Philosophy, 13(2), 41-56. Reprinted in Burgess (2008: 85-103). Citations to reprint.

Burgess, John P. (2008). Mathematics, Models, and Modality: Selected Essays. Cambridge University Press.

Cappelen, Hermann and Ernie Lepore (2007). The Myth of Unarticulated Constituents. In Michael O'Rourke and Corey Washington (Eds.), Situating Semantics: Essays on the Philosophy of John Perry (199-215). MIT Press.

Dasgupta, Shamik (2009). Individuals: An Essay in Revisionary Metaphysics. Philosophical Studies, 145(1), 35-67.

Diehl, Catharine (2017). Ontological Nihilism and Existential Commitment: Studies in Metametaphysics (Unpublished doctoral dissertation). Humboldt University.

Diehl, Catharine (2018). Haecceitism without Individuals. Unpublished 
manuscript.

Field, Hartry (1980). Science without Numbers: A Defense of Nominalism. Princeton University Press.

Fodor, Jerry (1990). A Theory of Content and Other Essays. MIT Press.

French, Rohan (in press). Notational Variance and Its Variants. Topoi.

Hattiangadi, Anandi (2007). Oughts and Thoughts: Skepticism and the Normativity of Meaning. Oxford University Press.

Hawthorne, John (O'Leary-) and Andrew Cortens (1995). Towards Ontological Nihilism. Philosophical Studies, 79(2), 143-165.

Ketland, Jeffrey (2015). Abstract Structure. Unpublished manuscript.

Kripke, Saul (1982). Wittgenstein on Rules and Private Language. Harvard University Press.

Kuhn, Steven T. (1983). An Axiomatization of Predicate Functor Logic. Notre Dame Journal of Formal Logic, 24(2), 233-241.

Lewis, David (1990). Parts of Classes. Blackwell.

Perry, John (1986). Thought without Representation. Proceedings of the Aristotelian Society Supplement, 60, 137-51.

Quine, Willard van Orman (1948). On What There Is. Review of Metaphysics, 2(5), 21-39.

Quine, Willard van Orman (1960). Variables Explained Away. Proceedings of the American Philosophical Society, 104, 343-347.

Quine, Willard van Orman (1971). Algebraic Logic and Predicate Functors. In Richard Rudner and Israel Scheffler (Eds.), Logic and Art: Essays in Honor of Nelson Goodman (214-238). Bobbs-Merrill. Revised and reprinted in Quine (1976: 283-307). Citations to reprint.

Quine, Willard van Orman (1976). The Ways of Paradox and Other Essays (2nd ed.). Harvard University Press.

Quine, Willard van Orman (1992). Pursuit of Truth (2nd ed.). Harvard University Press.

Rayo, Agustín (2007). Ontological Commitment. Philosophy Compass, 2(3), 428-444. Russell, Jeffrey Sanford (2018). Quality and Quantifiers. Australasian Journal of Philosophy, 96(3), 562-577.

Schaffer, Jonathan (2009). On What Grounds What. In David Chalmers, David Manley, and Ryan Wasserman (Eds.), Metametaphysics: New Essays on the Foundations of Ontology (347-383). Oxford University Press.

Strawson, P. F. (1959). Individuals. Meuthen.

Turner, Jason (2010). Ontological Pluralism. Journal of Philosophy, 107(1), 5-34.

Turner, Jason (2011). Ontological Nihilism. In Karen Bennett and Dean W. Zimmerman (Eds.), Oxford Studies in Metaphysics (Vol. 6, 3-52). Oxford University Press.

Turner, Jason (2012). Logic and Ontological Pluralism. Journal of Philosophical 
Logic, 41(2), 419-448.

Turner, Jason (2016). Can We Do Without Fundamental Individuals? No. In

Elizabeth Barnes (Ed.), Current Controversies in Metaphysics (24-34). Routledge. Williamson, Timothy (2007). The Philosophy of Philosophy. Blackwell.

Woods, Jack (2018). Intertranslatability, Theoretical Equivalence, and Perversion.

Thought: A Journal of Philosophy, 7(1), 58-61. 